\title{
Disputas narrativas y enseñanza de la historia: aportes de una reflexión teórica
}

Erinaldo Vicente Cavalcanti ${ }^{1}$

Universidad Federal del Sur y Sudeste de Pará, Brasil

Autor de correspondencia: ${ }^{1}$ ericontadordehistorias@gmail.com Recibido: 11 de diciembre de 2020 Revisado: 17 febrero de 20201 Aprobado: 24 de septiembre de 2021 Publicado: 23 de octubre de 2021

\section{Resumen}

En la ciencia histórica, la narrativa ha sido objeto de reflexión de diferentes autores, desde distintos enfoques. Este artículo tiene el fin de ampliar la reflexión sobre la narrativa histórica —académica y didáctica-, para comprender los límites de su pretensión de representar el pasado. Con esta problematización, el objetivo es poner la narrativa como foco de análisis en la enseñanza de la historia como una posible forma de enfrentar las disputas narrativas que son parte de la rutina del aula. La metodología parte del análisis crítico de literatura especializada, con el objetivo de entender en qué consisten los criterios que dan legitimidad a la narrativa histórica. En conclusión, con base en el marco teórico - especialmente en Paul Ricœur-, se argumenta que los procedimientos que constituyen la narrativa histórica pueden ser utilizados como una estrategia viable para tratar las disputas narrativas en el aula y promover la comprensión de la relación de confianza y credibilidad de que goza este informe escrito en la tarea para representar el pasado.

Palabras clave: historia, narrativa, enseñanza, representación, teoría 


\title{
Narrative disputes and history teaching: contributions from a theoretical reflection
}

\begin{abstract}
In historical science, narrative has been a subject of reflection by different authors, from different approaches. This article aims to expand the reflection on the historical narrativeacademic and didactic - in order to understand the limits of its attempt to represent the past. Based on this problematization, the objective is to set narrative as a focus of analysis in the teaching of history as a possible way to face the confrontation of narratives that are part of the classroom routine. The methodology is based on the critical analysis of specialized literature, seeking to understand the criteria that legitimize the historical narrative. In conclusion, based on the theoretical framework-especially on Paul Ricœur-it is argued that the procedures that constitute the historical narrative can be used as a viable strategy to cope with narrative disputes in the classroom and to promote the understanding of the relationship of trust and credibility that such written report holds in the task of representing the past.
\end{abstract}

Keywords: history, narrative, teaching, representation, theory

\section{Disputas narrativas e ensino de história: contribuições de uma reflexão teórica}

\section{Resumo}

Na ciência histórica, a narrativa tem sido objeto de reflexão de diferentes autores, a partir de diferentes abordagens. Este artigo visa ampliar a reflexão sobre a narrativa histórica - acadêmica e didática - para compreender os limites de sua intenção de representar o passado. Com esta problematização, o objetivo é estabelecer a narrativa como foco de análise no ensino da história como uma forma possível de enfrentar a disputa de narrativas que fazem parte da rotina da sala de aula. A metodologia é baseada na análise crítica de literatura especializada, buscando compreender os critérios que legitimam a narrativa histórica. Em conclusão, com base no quadro teórico - especialmente em Paul Ricœur - argumenta-se que os procedimentos que constituem a narrativa histórica podem ser usados como uma estratégia viável para enfrentar as disputas narrativas na sala de aula e para promover a compreensão da relação de confiança e credibilidade que tal relatório escrito tem na tarefa de representar o passado.

Palavras-chave: história, narrativa, ensino, representação, teoria 
En la actualidad, uno de los desafíos que enfrenta un profesor de historia o de disciplinas de humanidades en el ejercicio de su enseñanza son las llamadas guerras narrativas. Este concepto ganó fuerza en el campo dela enseñanza de historia y ciencias sociales, especialmente cuando el profesor e investigador canadiense Christian Laville (1999) publicó una reflexión importante sobre las disputas políticas más diversas en torno a la enseñanza de la historia. Él mostró cómo los enfrentamientos sobre la enseñanza de la historia tomaron forma y desencadenaron varias disputas, especialmente en el campo de las propuestas curriculares. Varios países han desarrollado luchas reales. Diferentes proyectos políticos han utilizado la fuerza y el poder para definir políticas públicas sobre qué y cómo enseñar en la historia y cuáles materiales didácticos debería ofrecer esa enseñanza. Como ha demostrado dicho autor, esas guerras no fueron solo narrativas, sino también políticas. Además, despertaron diferentes disputas en países como México, Brasil e Inglaterra, entre otros.

Esa guerra de narrativas se ha desarrollado de diferentes maneras. Los pasados son disputados en el presente por distintas narrativas, incluso las de los alumnos. Con el aumento del acceso a plataformas, aplicaciones y sitios web, ellos consumen una diversa cantidad de narrativas sobre el pasado histórico. En este sentido, parece que las disputas narrativas en el aula también pasan por la disputa sobre a qué pasados se accede y, sobre todo, cómo se validan y se reconocen como legítimos y confiables. En ese sentido, centrar la atención en la narrativa, como foco de análisis en la enseñanza de la historia, es una estrategia plausible para enfrentar dichas disputas en el aula.

La narrativa, especialmente la escritura, quizás sea el principal producto con el que establecemos diálogos. Producimos narrativas textuales en forma de trabajo de grado, de disertaciones y de tesis, en el ámbito estrictamente académico. Además, las fabricamos en forma de artículos, ensayos, libros y capítulos de libros académicos. También las producimos en análisis técnicos sobre proyectos, libros y artículos. En un porcentaje menor, los profesionales capacitados en esa área de conocimiento también escriben libros de texto en forma de narrativas. Por lo tanto, no es improbable decir que la narrativa es el producto principal por el cual una parte de nuestro trabajo está representado. También es a través de estas que establecemos diálogos con otros temas.

El debate sobre la narrativa ha sido parte del proceso por el cual la historia se convirtió en ciencia (Dosse, 2012). Algunos investigadores en diferentes momentos ya se han enfrentado al debate sobre la narrativa y la han problematizado desde diferentes perspectivas (Carr, 2016; Hartog, 2011; Ricour, 2007; White, 2019). En este texto, abordamos algunos de esos autores porque sus reflexiones ayudan a problematizar el tejido constitutivo de la narrativa historiográfica. De manera especial, los análisis de Ricœur $(2007,2010)$ ayudan a ampliar las posibilidades de aprehensión y uso de la narrativa como un producto fabricado en historia. Como esta ciencia tiene una estrecha relación con la enseñanza, las reflexiones de dichos autores también pueden contribuir al debate sobre la narrativa como un elemento abordado en la historia que se enseña en el aula de educación primaria y secundaria.

En este sentido, buscamos problematizar algunas dimensiones constitutivas de la 
narrativa histórica - académica y didáctica - cuando es entendida como una representación del pasado y una presentación del presente. Con las interpretaciones, el objetivo es analizar la narrativa como una herramienta por explorar en la enseñanza de la historia. La propuesta es revisar el tejido de la narrativa para que sea una estrategia viable en el aula.

Ubicar la narrativa como el principal objeto de reflexión en la enseñanza de la historia puede mejorar las experiencias de aprendizaje histórico en el aula. De esa manera, es una posibilidad de enseñanza y aprendizaje en la que los contenidos estudiados no se trabajen solo de manera expositiva. Trabajar con el contenido enseñado y explorar su constitución narrativa también puede contribuir a expandir la capacidad analítica de nuestros estudiantes.

Estudiar los contenidos y comprender el tejido constitutivo de las narrativas que los alcanzan en el presente y que están en los libros de texto puede ampliar la interpretación de los estudiantes, al hacerles cuestionar las diferentes narrativas con las que tratan a diario. También puede ayudarlos a comprender que los contenidos trabajados son, sobre todo, el resultado de una representación narrativa, así como de otros eventos.

\section{Narrativa histórica y representación del pasado}

Poner la narrativa en el centro de las discusiones en torno a la escritura de la historia no significa defender que la historia es una narrativa cualquiera, que se resume o es equivalente a un informe descriptivo. Por el contrario, se trata de pensar en el estado narrativo en la escritura de la historia en términos de composición, trama e intriga, que asignan significados a la historiografía. Es decir, "no se trata de defender una historia que es simplemente narrativa, sino de discernir el lugar apropiado de la historia entre las ciencias y sus condiciones de inteligibilidad, reconociendo la pertenencia de la historiografía al campo narrativo" (Ricœur, 2010, p. 14).

En ese sentido, la narrativa ocupa un lugar central, ya sea como producto intelectual del investigador -o profesor- o como informe de experiencias humanas sobre los temas de investigación o enseñanza. Es oportuno reforzar que solo tenemos acceso al pasado vivido por la humanidad a través de las diferentes narrativas construidas para servir a diferentes intereses - no solo los de la historia-. La narrativa es el vestigio que permite que las experiencias humanas crucen los límites del tiempo. Permite que lo sucedido en tiempos pasados se dé a conocer hoy y se proyecte para tiempos futuros.

En su libro clásico Memoria, historia, olvido, Ricœur (2007) elabora una densa reflexión sobre la dimensión narrativa de la práctica historiográfica. Para él, la representación del pasado que ha sido construida por la ciencia histórica se elabora a través de un procedimiento que articula las fases documental, explicativa/comprensiva y de representación, que se constituye en una narrativa escrita.

No se debe atribuir una relación complementaria entre la representación narrativa y el evento representado. La narrativa histórica no tiene una relación de causalidad o determinación con la experiencia representada, aunque tengan una conexión. La narrativa histórica no está determinada por el hecho narrado, aunque este último no pueda separarse de ella. Sin embargo, eso no significa que haya una relación de antinomia entre la narrativa histórica y la historia narrada. Como señaló Carr (2016), no hay divorcio entre la narrativa histórica y lo que se llama la realidad histórica representada por la narrativa. La narrativa 
histórica no es una construcción extraña apartada de la experiencia sobre la cual produce una historia. En otras palabras, la narrativa histórica y la historia narrada no son mutuamente excluyentes; son distintas, pero se necesitan para existir. Según Chartier (2009), la narrativa construida por la ciencia histórica no se confunde con la experiencia investigada, aunque no pueda existir sin ella.

En tal sentido, la narrativa histórica no es una copia del evento narrado. En esta línea de interpretación, Ricœur (2007) afirma:

En vano se busca un vínculo directo entre la forma narrativa y los eventos tal como tuvieron lugar; la conexión solo puede ser indirecta a través de la explicación y, debajo de esto, a través de la fase documental, que a su vez se refiere al testimonio y al crédito dado a la palabra de los demás. (p. 256)

Para el autor, es necesario establecer las distinciones entre narrativa y evento narrado y buscar conexiones entre la narrativa histórica, la fase explicativa/comprensiva y la fase documental.

La relación entre la narrativa producida por la ciencia histórica y la ficción también fue registrada por Certeau (2007). Para él, hasta cierto punto, "el pasado también es ficción del presente" (p. 21). En otras palabras, la narrativa fabricada por el historiador en el presente en cierta medida crea y representa el pasado, y el pasado se reinventa como creación/ficción en el presente por el historiador. Desde ese ángulo de percepción, Ricœur (2007) dictaminó: "considerado desde el ángulo de la imaginación del lenguaje, la narrativa histórica y la narrativa de ficción pertenecen a una misma clase, la de «ficciones verbales»" (pp. 263-264).

Sin embargo, este análisis no demuestra la inexistencia de diferencias entre la narrativa histórica y la narrativa de ficción. Es esencial comprender que son distintas. Como sostiene Pomian (2003), "no hay historia sin la conciencia de un límite entre el reino de la realidad y el reino en el que la ficción ejerce sus plenos poderes" (p. 11). Es esencial, por lo tanto, comprender las singularidades constitutivas de la narrativa histórica y no jerarquizar ni definir qué narrativa es fiel o verdadera. Así mismo, es fundamental comprender el proceso de construcción de la narrativa producida por la ciencia histórica para ampliar la reflexión sobre su intención de representar el pasado y, por ende, movilizarlo como una estrategia para el estudio y la enseñanza de la historia.

A través de ese camino analítico, comprender que hay elementos comunes entre la narrativa histórica y la ficticia no significa disolver las distinciones existentes. De igual forma, comprender que en la escritura producida por la ciencia histórica hay elementos comunes y presentes en otros géneros narrativos - especialmente en la ficción- no significa decir que las narrativas son equivalentes.

Las reflexiones de Ricœur $(2007,2010)$ sugieren una posible forma de enfrentar los límites de la representación del pasado construida por la narrativa histórica, en la que difiere de la narrativa ficticia. Estos análisis pueden ser abordados tanto en el debate específicamente académico como en las reflexiones desarrolladas en el aula, con respecto a la pretensión de la narrativa histórica de representar el pasado.

La narrativa histórica - académica o didáctica - es construida a partir de un conjunto de reglas que resultan en un texto escrito. Esas reglas están constituidas por normas 
institucionales que permiten ciertos tipos de narrativas y prohíben otros, como lo demuestra Certeau (2007). Las fases documental y de explicación/comprensión, para Ricour (2007), preceden y hacen posible la fase de representación, que produce una narrativa. Esas dos fases son inseparables y crean las condiciones que dan sentido y distinción a la narrativa histórica. Esta, entonces, es un producto textual que resulta de procedimientos verificables y que moviliza rastros documentales y procedimientos de explicación/comprensión que manifiestan una representación del pasado. Tal representación narrativa está constituida por normas y procedimientos reconocidos y validados por un cuerpo de intelectuales socialmente legitimados.

Así, no se deben buscar solo en la narrativa histórica —en la fase de representación narrativa; en el texto escrito- los elementos por los cuales el significado y el reconocimiento pueden atribuirse a la representación del pasado. Es necesario identificar y reconocer en la dimensión escrita de la narrativa histórica los elementos y procedimientos de las fases que precedieron y que acompañan simultáneamente su construcción textual.

En ese sentido, los procedimientos constitutivos de la narrativa histórica, en sus tres fases, establecen relaciones necesarias con los criterios que atribuyen legitimidad a la escritura histórica en su pretensión de representar el pasado. La referencialidad - por la que la narrativa histórica se distingue de la narrativa ficticia y disfruta del estatuto de reconocimiento- no reside en las figuras del discurso o en los recursos gramaticales movilizados en la construcción lingüística del discurso. Por lo tanto, en la dimensión del lenguaje no hay elementos ni procedimientos instituyentes a partir de los cuales la narrativa histórica busque representar el pasado.

\section{Guerras de narrativas y enseñanza: disputas por la historia}

¿Cómo lidiar con las disputas narrativas que ocupan un lugar cada vez más denso y tenso en las clases de historia? Decir que este o aquel texto es una narrativa histórica y que fue elaborado por un especialista ya no parece tener los efectos deseados. Ni registrar que una determinada narrativa está presente en los libros de texto de historia - y que, por lo tanto, es confiable - ni afirmar que determinada narrativa está presente en los libros académicos producidos por la ciencia histórica han producido los efectos necesarios para garantizar la fuerza en las disputas con otras narrativas.

En ese sentido, es posible que tengamos que ubicar la narrativa como un objeto central de reflexión en la clase de historia y, en consecuencia, promover una reflexión para presentar y comprender lo que finalmente produce y atribuye significado, reconocimiento y legitimidad a la narrativa histórica, ya sea en la versión científica o en la didáctica. Sin embargo, esa problematización no debe tener como objetivo poner a la narrativa histórica como la única capaz de representar el pasado.

En esa perspectiva, en nuestra experiencia, la producción y la comercialización de diferentes narrativas sobre los temas más diversos han aumentado. Nuestros estudiantes llegan cada vez más alimentados por diferentes narrativas producidas y ampliamente difundidas en redes sociales digitales. Por lo tanto, se propone ampliar el debate, ya que la debida claridad sobre los elementos constitutivos de la narrativa histórica es un camino que 
se puede tomar. Es importante movilizar la reflexión sobre los procedimientos mediante los cuales el reconocimiento y la legitimidad se atribuyen al informe narrativo producido por la historia. Ese recurso puede ser utilizado para tratar la diversidad de narrativas que nuestros estudiantes consumen, las cuales compiten con aquellas fabricadas por la ciencia histórica y entran en la disputa de representar el pasado.

Seguramente las narrativas producidas por las plataformas de entretenimiento y por las aplicaciones de comunicación son obras de ficción, incluso cuando representan algún evento histórico del pasado. Es importante que el maestro tenga claro que, aunque existe cierta proximidad entre narrativa ficticia y narrativa histórica, esos términos no son sinónimos. Aquí, una vez más, se puede recurrir a Ricœur (2007), que es categórico al afirmar que la narrativa histórica y la narrativa ficticia son géneros literarios distintos e incluso antinómicos. Para él, "una cosa es una novela, incluso una realista; otra cosa, un libro de historia. Se distinguen por la naturaleza del pacto implícito entre el escritor y su lector" (p. 274). El pacto a que se refiere el autor es una construcción cultural por la cual el lector, al abrir un libro de historia, desea encontrar una narrativa sobre un evento que realmente ocurrió. No es que esa narrativa debe ofrecer la descripción exacta de los hechos, sino que debe ser plausible, admisible, creíble y verificable. Como defiende Florescano (2016), es importante reconocer que la narrativa producida por la historiografía, aunque limitada, no es equivalente a la narrativa ficticia, ya que su elaboración resulta de una construcción académicamente rigurosa y conceptualmente controlada.

Esa reflexión de Ricour (2007) es importante porque una de las dimensiones que dan significado y reconocimiento a la narrativa histórica está en la relación que el lector —estudiante, maestro o cualquier otro ciudadano- construye con el texto escrito por la ciencia histórica. En otras palabras, no se trata solo de la estructura semántica, los argumentos utilizados, los documentos movilizados y las figuras del discurso activadas.

Los procedimientos por los cuales se produce la narrativa histórica permiten establecer una relación de confianza entre lo que se narra y la manera como es narrado. Esa relación de credibilidad no tiene como objetivo dar a la narrativa histórica el poder de representar los eventos tal como ocurrieron. Sin embargo, se presenta como una narrativa confiable, en la medida en que ofrece los elementos y procedimientos por los cuales se constituye. Es una narrativa verificable.

La relación de confianza puede fortalecerse en la medida en que quede claro que la narrativa historiográfica, en su versión académica o didáctica, no debe ser entendida como un reflejo de las experiencias reportadas. El pacto establecido y los sentidos estimulados no pueden proyectar una relación de correspondencia exacta entre el evento narrado y la representación narrativa elaborada en la escritura de libros didácticos o académicos.

La relación de confianza también puede fortalecerse al recurrir a cómo se aprende la escritura historiográfica, en su versión académica o didáctica, como una narrativa producida por normas y reglas reconocidas por un cuerpo de profesionales calificados. Es decir, no se construye sin procedimientos rigurosos. En el caso de la narrativa producida por el libro de texto, aún es necesario explicar algunas prácticas que singularizan y hacen posible que esta sea elaborada.

Es importante enfatizar que esa reflexión no tiene como objetivo transformar la clase de 
historia en una etapa de disputas para definir cuál narrativa es ficticia y cuál es histórica. Tampoco pretende dar a la narrativa ficticia el estado de un informe de fantasía, mientras se otorga a la narrativa histórica el lugar de la presentación de informes verdaderos e incuestionables ${ }^{1}$. Uno debe tratar de comprender el estado narrativo de la escritura histórica para comprender sus límites y posibilidades con respecto a su intención de representar el pasado y, por lo tanto, expandir su potencial para competir con otras narrativas. La afirmación de que una narrativa es confiable porque es producida por la ciencia histórica y que otra es falsa porque no es fabricada por la ciencia puede no ser el mejor argumento o camino para seguir.

Es importante tener en cuenta que problematizar la narrativa en las clases de historia no significa "perder el tiempo". El tiempo es un factor esencial para los profesores de historia en educación básica, especialmente con el vasto menú de contenidos para trabajar durante el año. La sugerencia es no trabajar la narrativa separada del contenido. La opción es trabajarla con el contenido, al problematizar la narrativa por la cual está representado. Lo que cambia es el camino y el ángulo de percepción. En lugar de ofrecer a los estudiantes una larga lista de temas distribuidos por una tabla de contenidos en los libros, a partir de una exposición casi siempre descriptiva, cronológica y lineal, se ofrecerá un menú sobre cómo se construyen esos contenidos, cómo se fabrican y se representan hasta su llegada al aula. Esa opción puede contribuir para que los temas estudiados no sean olvidados después de experimentados en forma de aprendizaje y/o actividades de evaluación. No se trata de hacer historiografía con los alumnos de educación básica, sino ampliar su comprensión sobre los límites de representación de la narrativa que se consume en la clase. Por lo tanto, se busca problematizar la forma de elaboración de los contenidos para ampliar la comprensión de cómo representan los pasados estudiados.

No se busca transformar a los jóvenes de la educación primaria y secundaria en pequeños historiadores, sino contribuir a la construcción crítica del mundo en el que viven: un lugar marcado por las experiencias de disputas narrativas cada vez más densas. La intención es expandir las capacidades cognitivas por las cuales los estudiantes consumen diferentes narrativas en su vida diaria. A medida que el estudiante tiene la oportunidad de estudiar los contenidos y de entender cómo se producen como una construcción narrativa, puede ampliarse su capacidad de problematizar las otras narrativas que consume a diario.

En ese sentido, dirigir el foco de atención a la narrativa puede hacer que el debate vuelva a comprender que una clase de historia es un acto narrativo, incluso cuando la clase se reduce a una exposición cronológica y se presenta a través de una narrativa que inventa, representa y presenta tiempos, espacios y personas. En esa perspectiva, Albuquerque Júnior (2019) es enfático y destaca que "olvidamos, tal vez, que enseñar es narrar, que la historia es una historia, que la historiografía es un género narrativo y, como el espacio del aula, depende de la narrativa para existir" (p. 223).

\section{Narrativa y libro de texto: diálogos y posibilidades}

La narrativa principal que se consume en el aula, en forma de contenido aprendido y enseñado, se sirve por medio de los libros de texto. La literatura especializada muestra que

\footnotetext{
1 Sobre las relaciones entre narrativa, acontecimiento, ficción y representación, ver los aportes de Burke (2011), Chartier (2009) y Ginzburg
} (2007). 
esa herramienta sigue siendo importante para el trabajo del maestro en el aula (Abud, 2007; Prats 2012; Rüsen, 1997). Además, autores como Choppin (2000, 2004), Ramírez (2003), Munakata (2012), Serrano (2013), Valcárcel (2016), Rocha (2018) y Martínez y Rubio (2018) destacan la importancia y complejidad del libro de texto como objeto de investigación. Bajo esa perspectiva, el libro de texto puede ser apropiado y abordado como una narrativa compleja por la cual ciertos pasados se producen, se presentan y se representan en forma escrita en nuestro presente. Sin embargo, atribuir al libro de texto de historia el nombre de narrativa compleja no significa que otras formas de narrativas históricas, ficticias o literarias sean menos o nada complejas. Con esa categorización solo queremos explicitar el proceso de producción de la narrativa didáctica. O sea, para comprender el libro de texto como una narrativa es necesario mostrar su camino de producción y enfatizar que es construida por diferentes profesionales de diferentes áreas, desde el profesor/investigador - que escribe el texto en la computadora-, hasta los profesionales en el campo del diseño gráfico, editores y revisores (Cavalcanti, 2016) ${ }^{2}$. También es esencial presentarles a los estudiantes las condiciones que hacen posible que se construya una narrativa particular, especialmente por medio de políticas públicas con las reglas y normas que condicionan las posibilidades de producción de los libros de texto.

Es importante que maestros y alumnos comprendan el conjunto de relaciones que permiten que se produzca dicha narrativa. Por lo tanto, es necesario presentar las etapas por las cuales la narrativa gana cuerpo y existencia; es decir, se deben mostrar los procedimientos relacionados con la producción textual y el uso de recursos documentales. Asimismo, es importante presentar, aunque rápidamente, los procedimientos y criterios de evaluación por los cuales pasan los libros de texto, que permiten la presencia de ciertas declaraciones en las narrativas didácticas, mientras que prohíbe otras. Además, es necesario mostrar los procedimientos para elegir los libros, desde el momento en que se aprueban hasta su llegada a la escuela.

De esa forma, la enseñanza de los contenidos en clase se debe realizar simultáneamente con la presentación del proceso de preparación. Al tiempo, hay que enseñar qué elementos y formas de presentación están contenidos en esa narrativa. Es decir, en lugar de ofrecer una narrativa solamente descriptiva de los contenidos, también se discute la presentación del proceso de su fabricación y la ruta de su preparación. La adición de esos "ingredientes" puede contribuir a una buena experiencia de enseñanza y potencializar el proceso de aprendizaje en las clases de historia. Por lo tanto, las reflexiones sobre la narrativa de un determinado contenido pueden ampliarse al explorar cómo los documentos son abordados y utilizados en la construcción de la escritura y cómo contribuyen a representar el evento narrado. Al tomar esa sugerencia como una posibilidad de trabajo, el maestro podrá elegir un contenido específico y estudiar qué documentos utilizaron los autores como fuente para elaborar las narrativas.

Una vez elegida esa propuesta, es posible comenzar el estudio del capítulo -o del contenido- a partir de una encuesta sobre los tipos de fuentes que los autores utilizaron para escribir la narrativa. En esa perspectiva, se identifican las fuentes que se usaron y el lugar que ocupan en el texto. El uso de la fuente en la producción textual se puede activar

\footnotetext{
2 Es muy importante que las discusiones se desarrollen durante el periodo de formación inicial para que los docentes puedan comprender el
} proceso de producción del libro y sus posibilidades de uso en el aula (Cavalcanti, 2019, 2020). 
para registrar las diferentes versiones del evento estudiado. Ese registro es importante para comprender cómo cada fuente utilizada puede mostrar de diferentes maneras ciertos aspectos o dimensiones sobre el mismo tema. En este sentido, vale la pena mencionar que las fuentes usadas no fueron producidas por los autores de la narrativa didáctica, ni fabricadas con el objetivo de servir como fuentes para un libro de historia. Muestran, por lo tanto, que la representación construida por la narrativa histórica no fue un invento de quien escribió el texto/libro.

Esa lectura puede ser demasiado obvia para profesores/investigadores. Sin embargo, muchos estudiantes de educación básica pueden preguntarse por qué la narrativa de los libros de texto es confiable y otra que circula en las redes sociales, por ejemplo, no lo es. Esa reflexión también se puede activar para mostrar que el mismo contenido estudiado tiene diferentes versiones, las cuales se pueden explorar para mostrar la importancia de reflexionar sobre las diferentes narrativas de los eventos y reforzar que es peligroso que haya una sola historia (Adichie, 2019).

Tales enfoques deben ser propuestos para que los estudiantes comprendan que los documentos abordados no son los eventos representados. Por lo tanto, para maestros y estudiantes, tan importante como usar la narrativa textual de los libros para hablar sobre un evento dado, lo es demostrar los procedimientos constitutivos por los cuales la narrativa fue producida, ganó materialidad y pudo ser consumida. En otras palabras, tan importante como saber lo que se narra en el libro es entender cómo se narra.

Para ampliar la comprensión de cómo la narrativa presenta y representa el contenido, una posibilidad viable es dirigir el ángulo de percepción hacia los elementos de su composición. Así, los caracteres semánticos y gramaticales son elementos importantes que permiten que la narrativa histórica, como construcción verbal, se configure de manera inteligible. Cuando se articulan adecuadamente, esos elementos permiten que la narrativa produzca una síntesis de lo heterogéneo a nivel de representación.

Armado con estas reflexiones, es posible dirigir el ángulo de percepción a ciertas áreas o a ciertos elementos del tejido de la narrativa didáctica. Como construcción verbal - de manera próxima a la perspectiva defendida por White (2019)_, es posible identificar en la narrativa histórica del libro de texto los temas de oraciones gramaticales, pronombres de tratamiento, inflexiones verbales y nominales, adjetivos y conjunciones para reflejar cómo la historia casi siempre se produce y se narra en género masculino. Los elementos gramaticales aparecen casi siempre flexionados para el sujeto masculino. Son, por lo tanto, indicativos de una forma de aprehender y de escribir historia. En el texto principal de la narrativa presente en los libros de texto, la inflexión verbal y nominal es predominantemente masculina. La representación producida por esa narrativa en los libros de texto también se puede explorar si se resalta la presencia del género masculino en los contenidos narrados. Al dirigir el ángulo de percepción a ese problema, es posible identificar qué sujetos aparecen como personajes en la representación. Así, pueden ser una interesante variable interpretativa la identificación y la catalogación de los personajes que aparecen representados en la narrativa didáctica. Al estudiar el feudalismo o la Grecia Antigua, por ejemplo, se puede tomar una ruta analítica que muestre qué personajes aparecen en la escritura, cómo aparecen y qué lugar ocupan en el evento narrado. El registro de quiénes son los sujetos en términos de 
edad, color, etnia y sexo también se puede agregar a la reflexión.

Identificar estos y otros elementos en la representación de las historias narradas puede y debe contribuir a repensar cómo se construye, se narra y se produce la historia que se enseña; qué visibilidades permite y qué discursos construye. Esas reflexiones también pueden contribuir a ampliar la comprensión de cómo los jóvenes o los ancianos son tratados por la historia, ya sea la escrita e investigada por la ciencia o la enseñada, la aprendida y la problematizada en el aula. Esa reflexión también se aplica al problema del color como elemento identificador de un tipo de raza o etnia en la historia. ¿Cómo se tratan a los niños, mujeres y hombres negros hoy día? ¿Qué lugar ocupan en las narrativas que describen los medios de comunicación más variados? Reflexionar sobre esos temas puede ampliar la comprensión del lugar ocupado por determinados grupos en las representaciones de las narrativas didácticas y en las relaciones cotidianas de la vida práctica.

Igualmente, es importante reflexionar sobre la figura de la mujer en las narrativas presentes en los libros de texto. Las mujeres casi siempre son invisibles, no solo en el lugar de autoría de la producción de las narrativas, sino también en las referencias gramaticales, cuando las expresiones semánticas se flexionan de acuerdo con el género masculino. Las mujeres son olvidadas como los personajes constitutivos de las historias narradas. Los eventos sobre los cuales se producen diferentes narrativas en los libros de texto son realizados por hombres. Problematizar las narrativas es ampliar la interpretación del lugar que las mujeres ocuparon en el tiempo. Es ampliar las posibilidades de cuestionar las funciones que se les asignan y, por lo tanto, reflexionar sobre los papeles desempeñados por mujeres y hombres en la vida práctica.

Esos temas también se pueden explorar en los documentos abordados en la narrativa textual de los libros de texto. Es decir, uno no solo debe cuestionar la ausencia o presencia de algunos elementos en el texto escrito del libro, sino también reflexionar sobre cómo aparecen esas dimensiones o temas en las fuentes documentales de cada capítulo. La exploración de las fuentes usadas en las narrativas didácticas permite ampliar las lecturas de los contenidos estudiados y mostrar cómo es posible producir diferentes narrativas sobre el mismo contenido.

Las fuentes documentales utilizadas en las narrativas textuales de los libros de texto también se pueden utilizar para registrar los procedimientos que dan confianza y reconocimiento a la narrativa histórica, porque muestran que esa fuente tiene valor documental. De esa manera, el recurso de la fuente documental se puede utilizar para mostrar y reforzar una de las fases que dan sentido a la narrativa histórica, llamada por Ricœur (2007) fase documental. Ese recurso contribuye a dar confianza y reconocimiento a la narrativa histórica. Es posible reforzar que el uso de fuentes documentales es parte de los procedimientos metodológicos y es reconocido y validado por los profesionales - docentes e investigadores- que trabajan en esa área del conocimiento.

En consecuencia, las fuentes no se insertan en las narrativas didácticas solo para ocupar espacio en el diseño del libro o para ilustrarlo - en el caso de una fuente fotográfica-. Se consideran registros producidos por las actividades de diferentes hombres y mujeres sobre el contenido estudiado y representado en la narrativa textual. Así, al hacer uso de las fuentes presentes en los libros de texto, los investigadores que escribieron la narrativa ofrecen al 
lector otros registros sobre el tema narrado. Esos registros son parte de los procedimientos metodológicos que la ciencia histórica toma como requisitos necesarios en el proceso de construcción de las narrativas, para que sean reconocidas y consideradas válidas.

Aun así, no es suficiente usar, mostrar o citar la fuente. Es necesario otro procedimiento metodológico: la explicación/comprensión que el autor de la narrativa textual debe elaborar, "cuando juntos, la escritura, la explicación integral y la evidencia documental probablemente acrediten la afirmación de la verdad del discurso histórico" (Ricœur, 2007, p. 292). En ese sentido, la fuente documental - que incluso puede ser un testimonio, un relato de memoria - se utiliza como la "materia prima" con la que el autor de la narrativa elabora y sostiene su argumento explicativo sobre el contenido narrado. De acuerdo con Ricœur (2007), "no tenemos nada mejor que el testimonio y la crítica del testimonio para dar crédito a la representación histórica del pasado” (p. 293).

Las fuentes apoyan la explicación, pues atribuyen un efecto de verdad a la narrativa escrita y no permiten que se elabore sin reglas, normas ni cuidados. Esos procedimientos requieren rigor $y$ aseguran que la narrativa tenga legitimidad.

Sin embargo, las reflexiones deben adaptarse adecuadamente a los objetivos que uno pretende lograr con la enseñanza de la historia. Después de todo, necesitamos tener claridad sobre lo que es enseñar la historia. Comprender los objetivos por alcanzar con la enseñanza de la historia en el aula de educación básica es una condición para problematizar la narrativa.

\section{Consideraciones finales}

San Agustín (2009) dijo que somos "seres temporales". También es posible afirmar que somos "seres narrativos". La narrativa en diferentes formatos, tipos, configuraciones y materialidades permite estudiar y aprender la historia. Nuestros estudiantes de diferentes niveles de educación "consumen" narrativas que no tienen reconocimiento ni legitimidad para representar el pasado. Sin embargo, decir que están equivocadas no es suficiente. Una estrategia es poner la narrativa en el centro de la reflexión para comprender cómo es su proceso de construcción y en qué consiste su representación sobre el pasado. Entender cómo se produce la narrativa, quién la produjo, dónde, por qué, para quién y para qué se construyó es un posible camino.

Como argumenta la bibliografía especializada, citada en este artículo, es solo por medio de la narrativa que se pueden conocer las experiencias de hombres y mujeres en el tiempo. En ese sentido, solo por medio de la narrativa conocemos prácticas, deseos y sueños de personas que vivieron en tiempos y espacios distantes y diferentes. La narrativa nos permite conectarnos entre tiempos muy distantes. Además, hace posible la comprensión entre espacios tan lejanos, entre personas tan diferentes y entre historias tan distintas y similares a la vez. Es solamente a partir de las narrativas que se puede aprender y enseñar esas y otras historias. Estas posibilitan conocer el mundo, nuestro mundo y otros mundos.

Por lo tanto, comprender cómo se construye la narrativa histórica, en la versión académica y/o didáctica, puede ser el camino que nos permita continuar creando, informando, representando, narrando y enseñando. Comprender y hacernos entender narrativamente - informar en qué consiste cada fase del proceso de la elaboración narrativa de la ciencia histórica- puede ser el mejor recurso para enfrentar las disputas de narrativas en tiempos 
marcados por las noticias falsas. Es posible afirmar que sin narrativa no hay historia ni se puede enseñar. Sin la narrativa no hay mundo ni humanos. Sí, la humanidad fue creada por la narrativa.

\section{Referencias}

Abud, K. (2007). A história de cada dia: saber escolar e saber acadêmico na sala de aula. En A. Monteiro, A. Gasparello, \& M. Magalhães (Orgs.), Ensino de história: sujeitos, saberes e práticas (pp. 107-177). Mauad X.

Adichie, C. (2019). O perigo de uma história única. Companhia das Letras.

Albuquerque Júnior, D. (2019). O tecelão dos tempos: novos ensaios de teoria da história. Intermeios.

Agustín, S. (2009). Confissões. Editora Vozes.

Burke, P. (2011). A história dos acontecimentos e o renascimento da narrativa. En P. Burke (Coord.), A escrita da História: novas perspectivas (pp. 335-356). Editora da UNESP.

Carr, D. (2016). A narrativa e o mundo real: um argumento a favor da continuidade. En J. Malerba (Coord.), História \& narrativa: a ciência e arte da escrita histórica (pp. 229-247). Vozes.

Cavalcanti, E. (2016). Livro didático: produção, possibilidades e desafios para o ensino de História. Revista História Hoje, 5(9), 262-284. https://doi.org/10.20949/rhhj.v5i9.219

Cavalcanti, E. (2019). Ensino de História, livro didático e formação docente de professores de História no Brasil. Enseñanza de las Ciencias Sociales, (18), 49-61. https://doi. org/10.1344/eccss2019.18.4

Cavalcanti, E. (2020). La formación docente inicial del profesor de Historia en Brasil: temas, reflexiones y desafíos. Ciencias Sociales y Educación, 9(18), 113-135. https://doi. org/10.22395/csye.v9n18a5

Certeau de, M. (2007). A escrita da história. Forense Universitária.

Chartier, R. (2009). A história ou a leitura do tempo. Autêntica.

Choppin, A. (2000). Los manuales escolares de ayer a hoy: el ejemplo de Francia. Historia de la Educación, (19), 13-37. https://revistas.usal.es/index.php/0212-0267/article/ view/10790

Choppin, A. (2004). História dos livros e das edições didáticas: sobre o estado da arte. Educação e Pesquisa, 30(3), 549-566. https://doi.org/10.1590/S1517-97022004000300012

Dosse, F. (2012). A História. UNESP.

Florescano, E. (2016). Para qué estudiar y enseñar la historia. Tzintzun. Revista de Estudios Históricos, (35), 135-146. http://www.tzintzun.umich.mx/index.php/TZN/article/ view/374

Ginzburg, C. (2007). O fio e os rastros: verdadeiro, falso, fictício. Companhia das Letras.

Hartog, F. (2011). Evidência da história: o que os historiadores veem. Autêntica. 
Laville, C. (1999). A guerra de narrativas: debates e ilusões em torno do ensino de História. Revista Brasileira de História, 19(38), 125-138. https://doi.org/10.1590/S010201881999000200006

Martínez, J., \& Rubio, J. (2018). Teoría y metodología de investigación sobre libros de texto: análisis didáctico de las actividades, las imágenes y los recursos digitales en la enseñanza de las Ciencias Sociales. Revista Brasileira de Educação, (23), 1-23. http:// dx.doi.org/10.1590/S1413-24782018230082

Munakata, K. (2012). O livro didático: alguns temas de pesquisa. Revista Brasileira de História da Educação, 12(3[30]), 179-197. https://www.periodicos.uem.br/ojs/index. $\mathrm{php} / \mathrm{rbhe} /$ article/view/38817

Pomian, K. (2003). História e ficção. Revista Projeto História, (26), 11-45. https://revistas. pucsp.br/index.php/revph/article/view/10532/7839

Prats, J. (2012). Criterios para la elección del libro de texto de historia. Íber: Didáctica de las Ciencias Sociales, Geografía e Historia, (70), 7-13. https://redined.mecd.gob.es/xmlui/ handle/11162/87471

Ramírez, T. (2003). El texto escolar: una línea de investigación en educación. Revista de Pedagogía, 24(70), 273-292. http://ve.scielo.org/scielo.php?script=sci_ arttext\&pid=S079897922003000200003\&lng=es\&tlng=es.

Ricœur, P. (2007). A memória, a história, o esquecimento. Campinas.

Ricœur, P. (2010). Tempo e narrativa. Vols. 1, 2 e 3. A intriga e a narrativa histórica. Martins Fontes.

Rocha, H. (2018). Desafios presentes nos livros didáticos de História: narrar o que ainda está acontecendo. Revista História Hoje, 7(14), 86-106. https://doi.org/10.20949/rhhj. v7i14.466

Rüsen, J. (1997). El libro de texto ideal. Reflexiones en torno a los medios de guiar las clases de historia. Íber. Didáctica de las Ciencias Sociales, Geografía e Historia, (12), 79-93. https://www.grao.com/es/producto/el-libro-de-texto-ideal

Serrano, S. (2013). Alfabetización histórica y competencias básicas en libros de texto de historia y en aprendizaje de los estudiantes. Revista Didáctica de las Ciencias Experimentales y Sociales, (27), 43-66. https://doi.org/10.7203/DCES.27.2648

Valcárcel, N. (2016). El uso del libro de texto de historia de España en Bachillerato: entre el aula y la casa. Revista História da Educação, 20(50), 69-93. http://dx.doi. org/10.1590/2236-3459/62453

White, H. (2019). Meta-história: a imaginação histórica do século XIX. Editora da Universidade de São Paulo. 